\title{
Network-wide MD and Network Coding for Heterogeneous Video Multicast
}

\author{
Ioannis Papadopoulos, Nikolaos Papanikos, Evangelos Papapetrou and Lisimachos Kondi \\ Department of Computer Science \& Engineering, University of Ioannina, e-mail: ipapadop,npapanik,epap,lkon@cs.uoi.gr
}

\begin{abstract}
Multiple Description coding (MD) has been proved to be very efficient for video multicast over random networks when combined with network coding. However, in the case where heterogeneous receivers are present in the network, the impact of the method is bounded by the fact that the solution to the inherent rate allocation problem is based on a compromise between low and high bandwidth receivers. To tackle this problem, we propose a novel video multicast scheme that enhances the combination of MD and network coding with a mechanism that allows all intermediate nodes to actively participate in MD coding by adjusting the size of the video descriptions. In this way, it is possible to tune the rate of the transmitted video stream in order to meet the needs of each receiver. The proposed method significantly improves the quality of the video delivered to all the receivers in the network, regardless of their heterogeneity.
\end{abstract}

\section{INTRODUCTION}

Reliable video multicast over lossy networks has received increased attention, especially after the proliferation of wireless networks. At the source side, the prominent method for resilience to transmission errors has been the use of Multiple Description (MD) coding [1]. Various researchers [2], [3] have combined MD with network-aware strategies that use the routing of video descriptions through different multicast trees in order to minimize susceptibility to transmission errors and maximize utilization of network resources. Network coding (NC) [4] is another alternative for error resilience and efficient resource utilization. The key idea is to allow the source and the intermediate nodes to code packets while the receiver is able to decode after receiving a set of encoded packets. Many researchers utilize network coding to provide reliable video multicast. Wang et.al. [5] proposed the combination of network coding with Scalable Video Coding (SVC). The combination of MD and network coding was first introduced in the MD/PNC method [6]. The authors first focused on the rate allocation problem, i.e. to optimize the rate of each description at the source in order to maximize video quality. Then, random linear network coding [7] is used to transmit the video descriptions. MD/PNC was refined [8] in order to provide heterogeneous multicast, i.e. support receivers with diverse connectivity characteristics. The basic idea is to collect feedback from all the receivers and optimize the rate of each description based on this information.

Although MD/PNC with receiver feedback improves the video quality on high and average bandwidth receivers, compared to a homogeneous multicast approach, this is done at the expense of the video quality experienced by low bandwidth receivers. This is because the rate allocation process is based on a compromise between high and low bandwidth receivers, i.e. video descriptions are determined in order to minimize the average video distortion. Such an approach may significantly deteriorate the video quality of low bandwidth receivers and limit the quality gain for high bandwidth receivers.

We take a more aggressive approach and propose a video multicast scheme that also utilizes MD and network coding. The novelty of the scheme is twofold. At the source, the rate allocation for each video description is optimized based on the feedback from the higher throughput receiver in order to provide the maximum possible video quality. Then, a rate-adaptation mechanism allows the intermediate nodes to actively participate in MD coding by reducing the rate of the video bitstream in order to accommodate the needs of heterogeneous receivers. We call the proposed method Network-wide MD/NC multicast $(N-M D / N C)$ and prove that it improves the video quality for high and low bandwidth receivers. To summarize, the contributions of this work are:

- a rate allocation process for MD that enables the maximization of video quality

- a novel approach that accommodates the needs of heterogeneous receivers by allowing intermediate nodes to participate in MD coding, thus transforming it from a source-centric operation to a network-wide one

The rest of this paper is organized as follows. In Section II, we provide a short background on MD and network coding while in Section III we formulate the rate allocation problem in MD. Then, in Section IV we delineate N-MD/NC, while its performance evaluation is presented in Section V. Concluding remarks are discussed in Section VI.

\section{PReliminaries}

\section{A. MD Coding Schemes}

The key idea in MD is the creation of video substreams of equal importance, called descriptions. Any description can be used for decoding, however video quality increases with the number of received descriptions. Priority Encoding Transmission (PET) [9] is one of the most important techniques used to produce video descriptions. Puri and Ramchandran [1] first followed such an approach to propose MD-FEC for video transmission over a lossy link. The video is usually partitioned in groups of pictures (GOP) and each GOP is processed separately. The bitstream resulting from a GOP is partitioned according to a set of $N$ rate breakpoints, $R_{1}, \ldots, R_{N}$. Note that the breakpoints denote the length of each part in bytes. However, since GOPs are created at a specific rate, the breakpoints also indicate the rate of the video, thus the term 


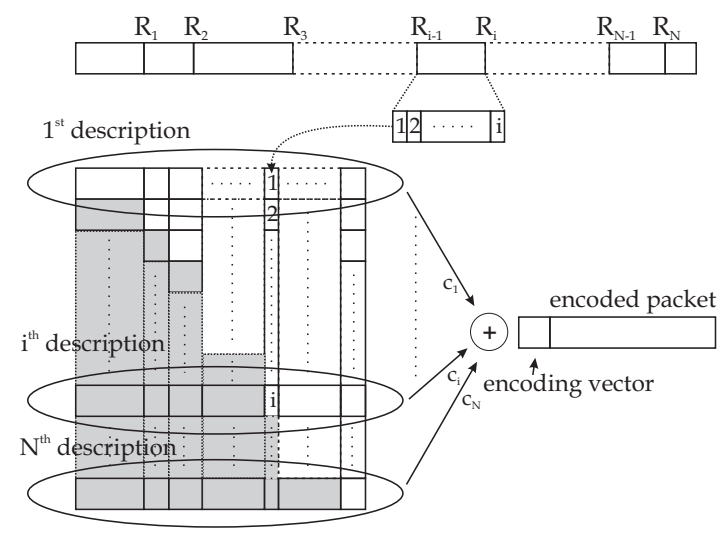

Fig. 1. Construction of descriptions in MD-based schemes and random linear coding (grey shaded parts are either parity or zero bits)

rate breakpoint. After the bitstream partitioning, the $i$ th part of the stream is divided into $i$ blocks and arranged vertically (fig. 1). Each of the $N$ descriptions consists of a block from each part of the bitstream along with parity bits from a FEC code and is transmitted as a packet in the network. Note that, given the GOP rate $R_{G}$, the size of a description is:

$$
l_{\text {desc }}=\frac{1}{N R_{G}} \sum_{k=1}^{N} \alpha_{k} R_{k}
$$

where $\alpha_{n}=N / n(n+1)$ [1]. A similar approach has been used in MD/PNC [6], [8] for multicast over a lossy network. The difference is that the parity bits are replaced with zero bits. Furthermore, each node transmits packets that are linear combinations of the $N$ descriptions. Those packets are created according to the principles of random linear network coding.

\section{B. Random Linear Network Coding}

The basic concept of network coding (NC) [4] is to allow source and intermediate nodes to code original packets, also called native, and transmit the encoded packets. A node is able to decode after receiving a sufficient number of encoded packets. NC provides improved resilience to errors by transmitting a slightly increased number of encoded packets. At the same time, it efficiently utilizes the network resources and achieves the flow of information between a source and the receivers at a rate equal to the multicast capacity [4]. Random Linear Network Coding (RLNC) is the most flexible way to construct a network code which is independent of the network topology [7]. The idea is to linearly combine packets based on the theory of finite fields and randomly select the required coefficients. More specifically, native packets are partitioned into symbols of size $s$. Then, an encoded packet is produced as a linear combination of $g$ native packets on the field $\mathbb{F}_{2^{s}}$ :

$$
p_{k}^{e n}=\sum_{i=1}^{g} c_{i} p_{i, k}^{n a t}, \forall k
$$

where $p_{k}^{e n}$ and $p_{i, k}^{n a t}$ are the $k$-th symbols of the encoded and the $i$-th native packet and $\left\langle c_{1}, c_{2}, \ldots, c_{g}\right\rangle$ is the set of the randomly selected coefficients, called encoding vector and appended to the packet header. Decoding of packets is performed when a node receives at least $g$ linearly independent encoded packets, also called innovative packets. Note that encoding at an intermediate node is possible without the need of decoding the native packets since a new encoded packet may be produced by linearly combining other encoded packets. Randomly selecting the vector $\left\langle c_{1}, c_{2}, \ldots, c_{g}\right\rangle$ is flexible for distributed implementations and it is also sufficient since the probability of producing linearly dependent combinations depends on the field size $2^{s}$ and can be negligible even for small values [10]. However, in order to practically implement RLC in networks, encoded packets need to be created over fixed groups of native packets, the so called generations [7].

\section{RATE Allocation IN MD MUlticAst}

The challenge in MD-based schemes is to optimally select the $N$ rate breakpoints $R_{1}, \ldots, R_{N}$, so that video quality is maximized. Traditionally, this problem has been formulated as an optimization problem with the objective to minimize a function of the distortion seen by the receiver/s. The problem has been solved for the trivial case where a link connects the source and the destination [1]. In the more complex case of multicast over a random network, MD/PNC [8] minimizes the average distortion seen by all the receivers, i.e.:

$$
D_{\text {avg }}=\frac{1}{M} \sum_{i=1}^{M} \sum_{k=0}^{N} p_{i}(k) D\left(R_{k}\right)
$$

where $M$ is the number of receivers, $p_{i}(k)$ is the probability that receiver $i$ obtains $k$ out of $N$ linearly independent packets and $D(R)$ is the distortion rate function for the source data. The constraints to the optimization problem are:

$$
R_{1} \leq R_{2} \leq \ldots \leq R_{N}
$$

which secures that the quality of the video increases with the number of received descriptions, and:

$$
\beta N^{2}+\sum_{k=1}^{N} \alpha_{k} R_{k} \leq \max _{i \in[1, \ldots, M]}\left\{m_{i}\right\}
$$

where $\beta\left(=8 R_{G}\right)$ is used to convert bytes/GOP to bits/sec. This last constraint mandates that the source rate should be less than the maximum max-flow bandwidth among the receivers. Note that the second term in the left-hand side of the equation represents the bitrate produced by the $N$ descriptions (eq. 1), while the first term accounts for the encoding vector bits.

\section{NeTwORK-WIDE MD BASED ON NETWORK CODING}

\section{A. Motivation and Basic Principles}

$\mathrm{MD} / \mathrm{PNC}$ [8] provided the evidence that the combined use of $\mathrm{MD}$ and RLNC is a successful methodology for video multicast in random networks. Therefore, we also adopt this approach. That is, MD is performed in each GOP to produce $N$ descriptions. Then, the descriptions are organized as a packet generation and the source node transmits $N$ linear combinations of the $N$ descriptions. Each intermediate node transmits an encoded packet for each innovative packet it receives. Besides the synergy of MD and network coding, this work focuses on the observation that the rate allocation method could be significantly improved. The reason is that, according to eq. 3, MD/PNC optimizes the average distortion seen by all the receivers. As a result, it is not possible for a 
high throughput node to receive the maximum possible quality because the rate allocation process also takes into account the delivery probabilities seen by poorly connected receivers. On the other hand, a low throughput receiver may not be able to keep up with the rate sent by the source, resulting in decoding failure. It will be shown later that the problem aggravates as the heterogeneity of the receivers increases. To tackle this problem, N-MD/NC takes a different approach in the rate allocation problem. The key concept is to allow the highest throughput receiver to receive the highest possible video quality. However, in order for the new rate allocation method to be meaningful, the multicast mechanism should provide a method for adjusting the video bitrate and/or trading video quality for packet redundancy so that lower throughput receivers can be supported. In the following we delineate the proposed multicast mechanism.

\section{B. Rate Selection at the source}

As discussed previously, we follow a different approach by seeking to minimize the distortion experienced by the receiver with the highest throughput. The rationale is that the highest possible video quality depends on the throughput and not the max-flow bandwidth. Therefore, we focus on the highest throughput receiver and let the other receivers tune the rate of the video stream through a network-layer mechanism (described in Section IV-D). Suppose that $m_{i}$ is the max-flow bandwidth of receiver $i$ and that $p_{e}^{i}$ is its the average loss rate. Then, we define the receiver with the maximum throughput:

$$
r=\underset{i \in[1, \ldots, M]}{\operatorname{argmax}}\left\{\left(1-p_{e}^{i}\right) m_{i}\right\}
$$

The rate allocation is performed in order to minimize:

$$
D_{r}=\sum_{k=0}^{N} p_{r}(k) D\left(R_{k}\right)
$$

which is the expected distortion at receiver $r$. The optimization is performed under the constraint in eq. 4 , while the constraint in eq. 5 is modified to account for $r$ 's max-flow bandwidth:

$$
\beta N^{2}+\sum_{k=1}^{N} \alpha_{k} R_{k} \leq m_{r}
$$

\section{Feedback mechanism}

In order to perform the aforementioned rate allocation process, the source needs the average loss rate $p_{e}^{i}$ and $p_{i}(k)$ for each receiver $i$ in order to choose $r$ and calculate $D_{r}$ respectively. Therefore, receivers are required to provide feedback information. To minimize the size of this information, it has been shown [8] that $p_{i}(k)$ can be approximated by the Binomial distribution $B\left(N_{i},\left(1-p_{e}^{i}\right)\right)$, where $N_{i}$ is the maximum number of linearly independent packets that node $i$ can receive for each GOP according to its max-flow bandwidth. Consequently, what receivers need to forward to the source is only the average loss rate, which can be calculated by observing the number of linearly independent packets received in every GOP [8]. The feedback can be provided on a periodic fashion. However, in order to reduce signalling, a triggered approach, i.e. send feedback when a change in average loss rate is observed, can be used. Furthermore, intermediate nodes should aggregate the received feedback information before forwarding it to the source node. Note that, in Section V, we simulate the required signalling and do not consider it negligible.

\section{Rate Adaptation Mechanism at Intermediate Nodes}

As mentioned previously, the proposed rate allocation mechanism is fine-tuned for the highest throughput receiver and ignores the other receivers. This strategy allows the video to be produced at the maximum possible quality and gives rise to the need for a rate adaptation mechanism in order to accommodate the needs of the other receivers. To this end, we make the observation that an intermediate node can reduce the video rate by pruning bits from descriptions. To understand this, recall that according to eq. 1 there is a close relation between the video rate and the description size. If a node receives

$$
l_{\text {desc }}^{i}=\frac{1}{N R_{G}} \sum_{k=1}^{i} \alpha_{k} R_{k}
$$

bytes from each of the descriptions, then it should be able to decode the video at rate $R_{i}$. Given the full size $l_{\text {desc }}$ of a description, an intermediate node can reduce the rate of the video bitstream to $R_{i}$ by pruning the last $l_{\text {desc }}-l_{\text {desc }}^{i}$ bytes. Note that this process is not obscured by network coding. Recall that the descriptions are linearly combined to produce encoded packets. According to eq. 2 , the linear combination is done in groups of $s$ bits (usually $s=8$ since the probability of producing linearly dependent combinations is negligible [10] for this value) therefore pruning the last $l_{\text {desc }}-l_{d e s c}^{i}$ bytes from an encoded packet is equivalent to the pruning of the same bytes from the native packets (i.e. the descriptions).

The rate-adaptation mechanism is implemented in every intermediate node. In this work we consider wireless networks where nodes usually have one interface. In the case of wired networks the mechanism should be implemented separately for each of the interfaces. An intermediate node $v$ needs to store the average loss rate for each downstream receiver, i.e. for each receiver from which $v$ received feedback information. Let $\mathcal{D} \mathcal{R}_{v}$ denote the set of receivers that are direct neighbors of node $v$, while $\mathcal{R}_{v}$ denotes the set of receivers with a distance greater than one hop from node $v$. In wireless networks it is possible that $\left|\mathcal{D} \mathcal{R}_{v}\right|>1$. Node $v$ can differentiate these two types of receivers by examining whether the received feedback information is aggregated or not. Let us define:

$$
p_{e}^{v}= \begin{cases}\max _{\forall i \in \mathcal{D R}_{v}}\left\{p_{e}^{i}\right\} & \text { if } \mathcal{D R}_{v} \neq \emptyset, \\ \min _{\forall i \in \mathcal{R}_{v}}\left\{p_{e}^{i}\right\} & \text { if } \mathcal{D} \mathcal{R}_{v}=\emptyset\end{cases}
$$

In other words, $p_{e}^{v}$ is the maximum average loss rate seen among the receivers in $\mathcal{D} \mathcal{R}_{v}$. If no such receiver exists then the minimum average loss rate seen among the receivers in $\mathcal{R}_{v}$ is used. If $B$ denotes the available bandwidth, then $v$ uses $B$ and $p_{e}^{v}$ to adjust the video rate. The rationale is that when neighboring receivers are present, $v$ should adjust the video rate to accommodate all receivers, including the one with the lowest throughput. On the other hand, when neighboring receivers are not present, $v$ adjusts the video bitstream by 
TABLE I

LOSS RATES FOR THE DIFFERENT EXPERIMENTS

\begin{tabular}{|c|c|c|c||c|c|c|}
\hline & No.1 & No.2 & No.3 & No.4 & No.5 & No.6 \\
\hline \hline$p_{I 1}$ & 0.08 & 0.08 & 0.45 & 0.45 & 0.45 & 0.45 \\
\hline$p_{I 2}$ & 0.18 & 0.78 & 0.95 & 0.58 & 0.76 & 0.87 \\
\hline$p_{I 4}$ & 0.18 & 0.78 & 0 & 0 & 0 & 0 \\
\hline$p_{R 2}$ & 0 & 0 & 0.24 & 0 & 0 & 0 \\
\hline \hline$R_{1} / R_{2}$ & $0.1 / 0.2$ & $0.1 / 0.8$ & $0.1 / 0.9$ & $0.5 / 0.6$ & $0.5 / 0.7$ & $0.5 / 0.8$ \\
\hline
\end{tabular}

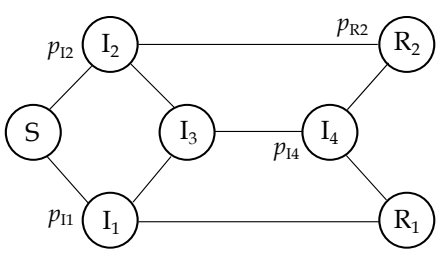

Fig. 2. The reference topology used in the experiments

taking into account the receiver with the highest throughput. This is because it is clear that the presence of lower throughput receivers in $\mathcal{R}_{v}$ is not due to the error rate in the local link. Depending on the values of $B$ and $p_{e}^{v}$, node $v$ may: a) adjust the video bitrate to the available bandwidth $B$, and/or b) trade bitrate for packet redundancy to compensate for increased packet loss rate. More specifically, we identify two cases:

i) $p_{e}^{v}>p_{e}^{r}$ : In this case, $v$ should transmit more packets $\left(N^{\prime}\right)$ in order to compensate for the increased error rate. Keeping in mind that the Binomial distribution is a good approximation for modeling the delivery of video packets in a random network through multicast [8], we define:

$$
N^{\prime}=\frac{1}{1-\left(p_{e}^{v}-p_{e}^{r}\right)} N
$$

However, increasing the number of transmitted packets comes at the cost of decreasing the rate of the video stream due to the bandwidth limitation. In more detail, $v$ chooses the maximum rate $R_{w}$ for which:

$$
N^{\prime}\left(\beta N+\frac{1}{N} \sum_{k=1}^{w} \alpha_{k} R_{k}\right) \leq B
$$

and through eq. 9 calculates the new size of descriptions.

ii) $p_{e}^{v} \leq p_{e}^{r}$ : In this case, there is no need for transmitting extra packets. However, if $B<m_{r}$ the rate of the video bitstream should be adjusted. The process followed is similar to the one in eq. 12 for $N^{\prime}=N$.

Note that, node $v$ should be aware of $p_{e}^{r}, m_{r}$ and the values $R_{i}, \forall i$ in order to perform the aforementioned process. This information can be conveyed through the packet's header.

\section{Performance Evaluation}

To evaluate the performance of $\mathrm{N}-\mathrm{MD} / \mathrm{NC}$, we used a wireless network with the well-known butterfly topology [4] with two receivers (fig. 2). This topology provides multiple paths to the receivers and intermediate nodes with only neighboring receivers $\left(\mathcal{D} \mathcal{R}_{v} \neq \emptyset\right.$ and $\left.\mathcal{R}_{v}=\emptyset\right)$, only non neighboring receivers $\left(\mathcal{D R} \mathcal{R}_{v}=\emptyset\right.$ and $\mathcal{R}_{v} \neq \emptyset$ ), or both types of receivers. Furthermore, the nature of wireless links renders this topology highly challenging for $\mathrm{N}-\mathrm{MD} / \mathrm{NC}$ because the actions of an intermediate node may have an impact on both receivers as well as other intermediate nodes. This limits the ability of a node to adjust its actions to each receiver separately. We conducted six experiments with different loss
TABLE II

AVERAGE PSNR SEEN BY $R_{1}$ AND $R_{2}$ FOR MD-PNC AND N-MD/NC

\begin{tabular}{|c|c||c|c||c|c|}
\hline \multirow{2}{*}{$\begin{array}{c}\text { Exp. } \\
\text { no. }\end{array}$} & $\begin{array}{c}\text { Loss Rate } \\
\left(R_{1} / R_{2}\right)\end{array}$ & \multicolumn{2}{|c|}{$R_{1}$} & \multicolumn{2}{c|}{$R_{2}$} \\
\hline \hline 1 & $0.1 / 0.2$ & 40.41 & $\mathbf{4 1 . 1 6}$ & 40.22 & 40.28 \\
\hline 2 & $0.1 / 0.8$ & $\mathbf{3 5 . 4 8}$ & 34.42 & 32.80 & $\mathbf{3 4 . 1 1}$ \\
\hline 3 & $0.1 / 0.9$ & 38.26 & 38.37 & 13.74 & 13.74 \\
\hline 4 & $0.5 / 0.6$ & 37.50 & 37.55 & 37.36 & 37.40 \\
\hline 5 & $0.5 / 0.7$ & 34.67 & 34.97 & 33.94 & 33.65 \\
\hline 6 & $0.5 / 0.8$ & 41.27 & 41.18 & 13.74 & $\mathbf{3 3 . 1 6}$ \\
\hline
\end{tabular}

rates at the receivers $R_{1}$ and $R_{2}$. In all experiments, $R_{1}$ is the highest throughput receiver while $R_{2}$ always experiences lowest throughput. In the first three experiments (no.1-3), the loss rate at $R_{1}$ is relatively low, while the loss rate at $R_{2}$ increases gradually. In experiments no.4-6, again we capture the performance of $\mathrm{N}-\mathrm{MD} / \mathrm{NC}$ when the heterogeneity in loss rate increases. However, in this case, the scenario is more challenging since the loss rates are relatively high for both receivers. To produce the desired loss rate at each receiver we introduced loss rates at intermediate nodes as shown in fig. 2. Table I reports those loss rates for each experiment as well as the loss rates seen at the receivers. In the first three experiments, in order to keep low the loss rate of $R_{1}$, we had to introduce a loss rate not only at $I_{1}$ and $I_{2}$ but also at $I_{4}$ or directly at node $R_{2}$. In the rest of the experiments, there is no such need since the loss rate of $R_{1}$ is relatively high. Therefore, the loss rate is introduced only at $I_{1}$ and $I_{2}$. Finally, the bandwidth of the wireless links was set to $2 \mathrm{Mbps}$. We implemented N-MD/NC as well as MD/PNC in ns2 [11]. More specifically, MD was implemented as an application layer agent, while the rate-adaptation mechanism as well as random linear coding were implemented as a routing agent. We executed 30 simulation runs for each experiment and report average values. The Foreman clip (CIF, 30fps, 288 frames, 16 frames/GOP) was used. The video is encoded with the MC-EZBC encoder [12], using $N=64$ descriptions. In order to calculate the distortion rate function $D(R)$, we encoded the video at different rates and recorded the corresponding distortion. Then, we used $D(R)=\alpha e^{-\beta R}$ as the fitting curve [13] with $\alpha=188.4$ and $\beta=8.777 \cdot 10^{-6}$.

Table II reports the average Peak Signal to Noise Ratio (PSNR) at the two receivers for N-MD/NC and MD/PNC. The PSNR is calculated based on the expected video distortion, i.e. $P S N R=10 \log _{10}\left(255^{2} / \bar{D}\right)$. The results of the first experiment confirm the correctness of the approach taken by $\mathrm{N}-\mathrm{MD} / \mathrm{NC}$. The modified rate-allocation mechanism manages to provide a quality improvement of $0.75 \mathrm{~dB}$ to $R_{1}$ compared to MD/PNC. On the other hand, the rate adaptation mechanism effectively adjusts the video rate to meet the requirements of $R_{2}$. As a result, $R_{2}$ receives virtually the same video quality (improvement of $0.06 \mathrm{~dB}$ ) with that received when MD/PNC is used. Fig. 3(a) illustrates the PSNR for each GOP of the video. Clearly, N-MD/NC improves the quality of the video received by $R_{1}$. At the same time, the video quality offered to $R_{2}$ is virtually the same to the quality offered by MD/PNC not only to $R_{2}$ but also to $R_{1}$. In the second experiment, we increase the difference in the loss rate of $R_{1}$ and $R_{2}$. N$\mathrm{MD} / \mathrm{NC}$ manages an improvement of $1.31 \mathrm{~dB}$ to the PSNR of 


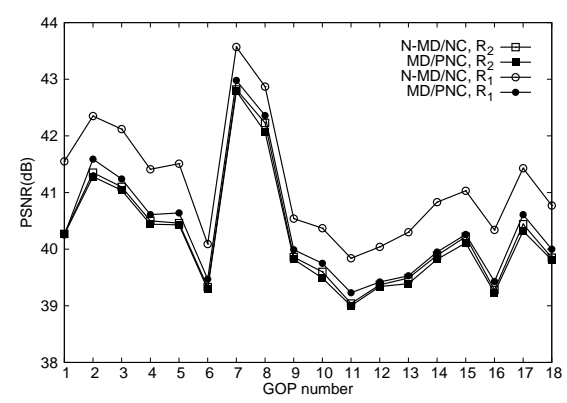

(a)

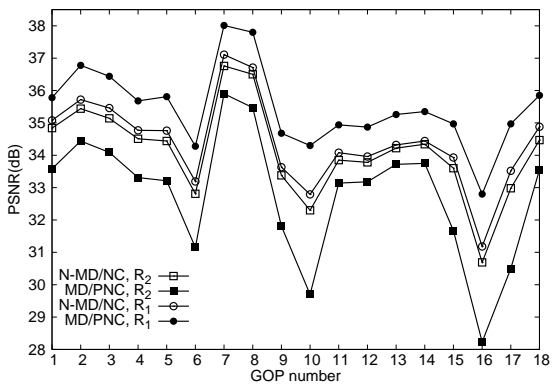

(b)

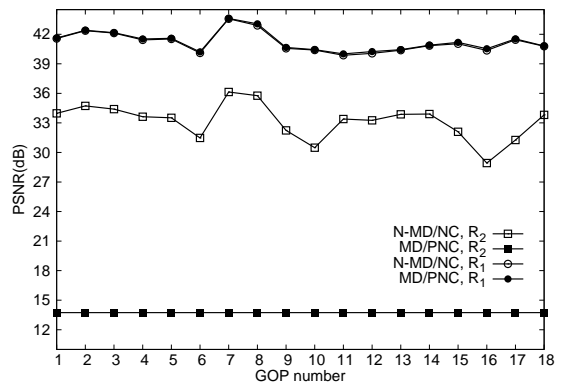

(c)

Fig. 3. PSNR for each GOP in experiment: (a) no. 1, (b) no. 2 and (c) no. 6

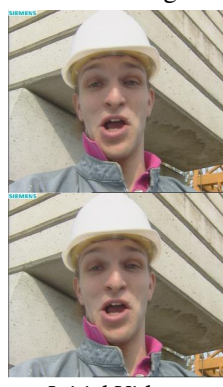

Initial Video

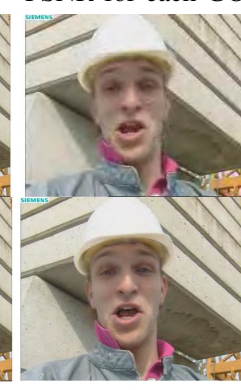

$\mathrm{N}-\mathrm{MD} / \mathrm{NC}\left(\mathrm{R}_{1}\right)$

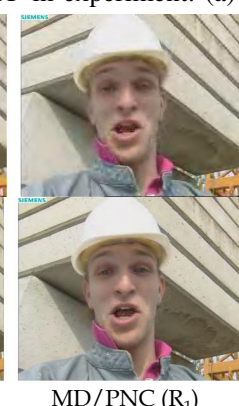

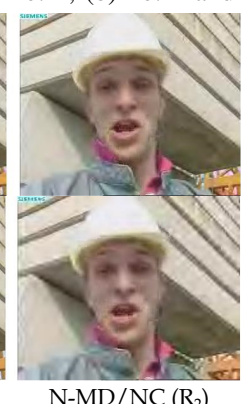

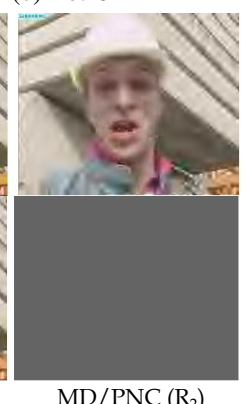

Fig. 4. Video snapshots as seen by the two receivers (image no.2 from GOP n.6). (Top): exp. no. 2 and (Bottom): exp. no. 6

$R_{2}$. However, concerning $R_{1}$, MD/PNC is better by $1.05 \mathrm{~dB}$. This is because the rate adaptation mechanism, performed at nodes $I_{2}$ and $I_{4}$, affects $R_{1}$ due to the nature of wireless transmission. Nevertheless, it is worth noting that improving the PSNR of poorly connected receivers is more important than doing so for high throughput receivers. To understand this, fig. 4 (top sequence of images) depicts snapshots taken from the received video at $R_{1}$ and $R_{2}$ for the two methods. The improvement in $R_{2}$ is obvious while no significant difference is witnessed in the snapshots from $R_{1}$. Finally, fig. 3(b) illustrates the PSNR for all GOPs. It is clear that the proposed method handles heterogeneity in a more efficient way than $\mathrm{MD} / \mathrm{PNC}$. To highlight this last conclusion, in experiment no.6, we consider a case of relatively high loss rates and significant heterogeneity in the two receivers. In this case, $\mathrm{MD} / \mathrm{PNC}$ fails to provide a reasonable video quality to node $R_{2}$ (PSNR=13.74dB). The reason is that the average distortion seen by both receivers (eq. 3) is not at all representative of the distortion seen in $R_{2}$. On the contrary, the PSNR of the video delivered to $R_{2}$ is $33.16 \mathrm{~dB}$ when N-MD/NC is used. It is interesting that this is accomplished without sacrificing the quality of $R_{1}$ 's video. This is also confirmed by fig. 3(c) and by fig. 4 (bottom sequence of images). The rest of the experiments also confirm the aforementioned conclusions. Note that the capacity of $\mathrm{N}-\mathrm{MD} / \mathrm{NC}$ to serve heterogeneous receivers is also limited. For example, in experiment no. 3 both schemes fail to deliver the video to $R_{2}$. However, N-MD/NC only fails in cases of extreme heterogeneity as the one in experiment no.3.

\section{CONCLUSION}

The combination of MD and network coding has been proved to be very efficient in video multicast over random networks. However, supporting heterogeneous receivers cannot be accomplished only through optimizing the description rates at the source node. To this end, we proposed a mechanism that efficiently adjusts the rate of the video bitstream at intermediate nodes in order to accommodate users with high heterogeneity. The proposed algorithm has been found to improve the video quality even in the challenging context of a wireless multihop network.

\section{REFERENCES}

[1] R. Puri and K. Ramchandran, "Multiple description source coding using forward error correction codes," in Signals, Systems, and Computers, 1999. Conference Record of the Thirty-Third Asilomar Conference on, vol. 1, 1999, pp. 342-346 vol.1.

[2] N. Sarshar and X. Wu, "Rate-distortion optimized multimedia communication in networks," in Proc. SPIE 6822, Visual Communications and Image Processing, 2008, p. 682206.

[3] V. Padmanabhan, H. Wang, and P. Chou, "Resilient peer-to-peer streaming," in Network Protocols, 2003. Proceedings. 11th IEEE International Conference on, 2003, pp. 16-27.

[4] R. Ahlswede, N. Cai, S.-Y. Li, and R. Yeung, "Network information flow," Information Theory, IEEE Transactions on, vol. 46, no. 4, pp. 1204-1216, Jul 2000.

[5] H. Wang, S. Xiao, and C. C. J. Kuo, "Robust and flexible wireless video multicast with network coding," in GLOBECOM, 2007, pp. 2129-2133.

[6] A. K. Ramasubramonian and J. W. Woods, "Video multicast using network coding," in Proc. SPIE 7257, Visual Communications and Image Processing, 2009, p. 72570E.

[7] P. Chou, Y. Wu, and K. Jain, "Practical network coding," in Proceedings of the Annual Allerton Conference on Communication Control and Computing, vol. 41, no. 1, 2003, pp. 40-49.

[8] A. K. Ramasubramonian and J. W. Woods, "Md/pnc with feedback for heterogeneous video multicast in lossy networks," in Proc. SPIE 7882, Visual Information Processing and Communication II, 2011, p. 78820M.

[9] A. Albanese, J. Blomer, J. Edmonds, M. Luby, and M. Sudan, "Priority encoding transmission," Information Theory, IEEE Transactions on, vol. 42, no. 6, pp. 1737-1744, 1996.

[10] C. Fragouli, J.-Y. Le Boudec, and J. Widmer, "Network coding: an instant primer," SIGCOMM Comput. Commun. Rev., vol. 36.

[11] S. Mccanne, S. Floyd, and K. Fall, "ns2 (network simulator 2)," http://www-nrg.ee.lbl.gov/ns/.

[12] Y. Wu, K. Hanke, T. Rusert, and J. Woods, "Enhanced mc-ezbc scalable video coder," Circuits and Systems for Video Technology, IEEE Transactions on, vol. 18, no. 10, pp. 1432-1436, 2008.

[13] Y. Chen, B. Wang, and K. J. R. Liu, "Multiuser rate allocation games for multimedia communications," Multimedia, IEEE Transactions on, vol. 11, no. 6, pp. 1170-1181, 2009. 